\title{
Counting Books and Beyond: Some Mathematical Books for Children
}

\section{Edmund Harriss}

One of the big challenges that faces people who love mathematics is how to pass that love onto our children, especially the beauty and creativity of the subject. In the equivalent situation, for those who love reading and literature, there is some clear advice: read to your children and help them find books that inspire. Perhaps that is a good place to start for mathematics as well.

The last decade or so has seen an amazing flow of accessible math books in general, speaking to quite a hunger for mathematical topics in a wide range of audiences. It is, therefore, no surprise that there are many possibilities aimed at children. MSRI, in collaboration with the National Councils for both Mathematics and English and the Children's Book Council, began awarding the Mathical Book Prize in 2015 to books written for children that "inspire a love of mathematics in the everyday world." The Mathical Book Prize web page (http://www.mathica1.org/) shows the rich range of books available, but here are some highlights, with rough age ranges given, though these are quite flexible. Excitement with the material is generally the best guide.

\section{CRASH BOOM! A Math Tale}

2019 Mathical winner, Ages 1-4

Robie H. Harris (Author), Chris Chatterton (Illustrator)

Candlewick 2018

This is the math tale of a slightly clumsy baby elephant playing with its wooden blocks. The elephant's goal is to

Edmund Harriss is a clinical assistant professor in mathematical sciences at the University of Arkansas. His email address is eharriss@uark.edu.

For permission to reprint this article, please contact: reprint-permission Qams.org.

DOI: https://dx.doi.org/10.1090/noti2269 make a tower of blocks "as tall as me." In the process it needs to count the blocks up and try to avoid the resulting towers from collapsing (the "crash boom!" of the title).

This book is related to the counting book, a classic genre of books for children, and certainly the most mathematical. Harris is thoughtful in her approach: the numbers are always counting the same thing (the blocks) and also serve as a key part of the story (trying to build the tower). The vocabulary of counting is thus matched to its meaning. When we become comfortable with the abstraction of numbers, we happily teach counting by presenting posters and books with examples like: one apple, two bananas, three oranges, thinking about the numbers and not the fruit. Thinking about numbers independent of context is an important aspect of learning mathematics, but it can be confusing to learners, especially when moving from counting to starting arithmetic. One apple and two bananas are certainly not three oranges, so the example above does not introduce this addition well. Learning the simple progression of the number names, without thought about units can thus obscure the ways that numbers relate to each other through addition and multiplication.

In addition to the counting of blocks, the book manages to hint at many other mathematical ideas, such as the notion of inequalities (the towers being taller or shorter than the elephant). Wooden blocks are also introduced (with lots of options of how to build towers) helping to develop thinking around a classic toy, which can be a great place to explore number and shape in a world of imagination. 


\section{Baby Goes to Market}

2018 Mathical winner, Ages 2-5

Atinuke (Author), Angela Brooksbank (Illustrator)

Candlewick 2017

Another thoughtful take on early counting is Baby Goes to Market, set in a market in Nigeria. The baby of the title is on its mother's back, and at the market is presented with six bananas, eats one, and places the remaining five into mother's basket. At the next stall there are five oranges, one of which is eaten and the remaining four placed in the basket, and you can probably see where this is going. The counting here is a little more direct, although the numbers are presented in the reverse order, with each connected directly to the next. The five remaining bananas can be directly compared to the five oranges from the next stall. This does present differing objects for each number, but the ritual of eating one and then noting how many remain demonstrates the connection between the numbers counting backwards, thus emphasizing the numbers separate from context.

An important aspect of this book is the cultural setting in Africa. In addition to helping show the beauty and wonder of mathematics to children, it is also essential to break down (or avoid creating) the cultural and gender barriers that too often keep people away from the subject. A part of this context helps those who identify with the characters and contexts see themselves in mathematics. Another significant part is the stereotypes and identification of scientists and mathematicians that all children are creating as they learn the subject. It does not matter if you identify as a mathematician if you are required to justify that identity in ways others (especially White men) are not. The introduction of mathematics to all children is perhaps the easiest and best time to attack these barriers. A book showing a group of African people having fun with counting, while wearing a gorgeous array of patterned clothing, is not a bad place to start.

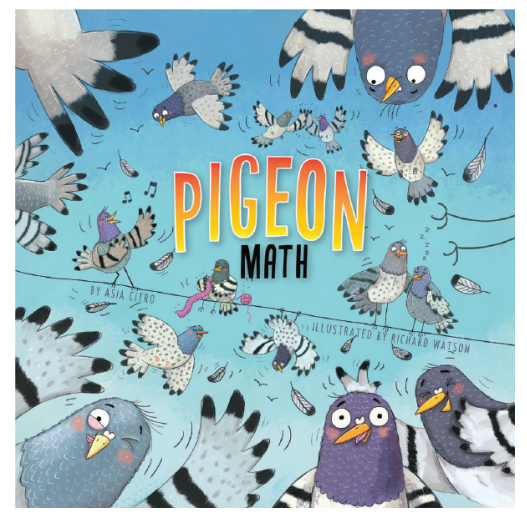

\section{Pigeon Math}

2020 Mathical winner,

Ages 3-7

Asia Citro (Author),

Richard Watson

(Illustrator)

The Innovation Press

2019

Pigeon Math moves beyond counting to arithmetic, as the narrator desperately tries to start their story about the collection of pigeons on a wire. The pigeons are not behaving and keep shifting around, leading to a bunch of different calculations of their numbers, as the day slowly passes. It seems that we never get to the original story that the narrator was going to tell, but there are plenty of other adventures in trying to tell it.

This approach again places the numbers into a consistent context so their behavior can start to be abstracted. In particular the appearance of "one cat" provides drama to the story while also showing that numbers can be used to count (and do arithmetic on) different objects, not just pigeons.

\section{Which One Doesn't Belong? \\ 2017 Mathical winner, Ages 4-10 \\ Christopher Danielson \\ Stenhouse Publishers 2016}

With really only one question, which is already given in the title, and very few other words, this introduction to mathematical thinking is quite different from what you expect from most books. It is focused on shape and geometry rather than number. On each page the reader is presented with four images and the constant question "Which one doesn't belong?" There is no one right answer, and certainly no wrong ones, in contrast to most mathematical textbooks and the familiar Sesame Street version of this question. The challenge is to say why the answer you chose was correct. For true masters of the form, a further challenge is to find reasons for all four possible answers. This simple idea is a work of genius, as the consistent question naturally leads to the identification of certain aspects of the images and the need for language to discuss the distinctions being made. Depending on the patterns these definitions might even shift. For example, do the "edges" of a shape have to be straight or can they be curved? If counting edges to identify three of the images and leave out the fourth, both definitions might be useful at different times.

The importance here is that the mathematical language developed must be precise but also creative and flexible. The viewer must communicate the ideas that occur to them, not something predefined. The precision of the language thus becomes part of the act of communication, rather than a way for the discussion of an idea to be wrong. As the book presents a carefully curated series of challenges, there are plenty of examples of geometric vocabulary that can be developed and discussed. The ability to present your own answers provides an opportunity to model the expository value of more standard mathematical language. That standard language then becomes something agreed upon for its utility, rather than simply insisted on.

This book presents a distinctly mathematical answer to the challenge I started with. Danielson has created a mathematical equivalent of reading to your child. The great number of possible justifications for each answer, and thus the repeatability of the book, could easily turn it into a regular ritual filled with creativity and the joy of discovery. 
The book How Many? by the same author has a similar format and provides his version of the counting book. It presents pictures of different objects (some cut up) again with the title providing the only question. The game here is to identify something in the image to count, and for anyone else involved to try to guess what you are talking about, just from the count you give them. Again, a lot of sophisticated and creative play can come from what can be considered "the same" and thus counted. Repeatedly using the exercise can lead to some curious and deeply mathematical places.

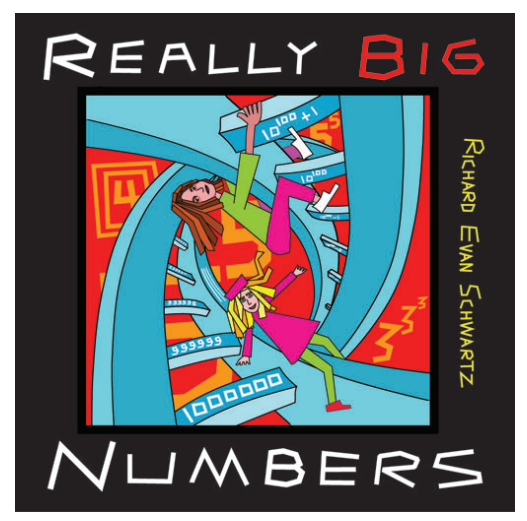

Really Big Numbers

2015 Mathical winner, Ages 6-?

Richard Schwartz

AMS 2014

One of the joys of talking about mathematics to other mathematicians is when you glimpse how they perceive the simplest objects of the subject, and how those understandings step up in a single story to the boundaries of current understanding. In a way this book is a counting book through that mathematician lens. Rich Schwartz puts together a ladder (his concept) of numbers starting with counting and repeatedly getting to numbers so large everything discussed before seems to disappear. There are some great facts along the way. Monkeys have about 100,000 hairs, but you would need about a trillion grains of sand to bury your father at the beach. Further on, the number of particles in the universe and the possible routes for a travelling salesman are quickly left behind as larger numbers are encountered. Even the googolplex is passed not much over halfway through the book.

The content of this book is approachable (and illustrated with colorful, engaging, and often surreal images), but the way the concepts expand with the numbers means that many readers will get stuck on the first read through, something the book addresses directly. From a meta point of view this introduces the idea that mathematical ideas build on mathematical ideas and we all have to sometimes sit with a level before working out how to go further. Learning to be comfortable in confusion and slowly sit exploring is one of the less-mentioned skills essential to many mathematicians.

This book provides a nice contrast to Which One Doesn't Belong? Both deal with mathematical play, with WODB giving a version of open-ended and creative exploration, whereas this book provides a map and tour notes from a previous traveler to explore deeper. Between them, these approaches provide a very interesting model to corrupt people into the joys of mathematics.

\section{The Miscalculations of Lightning Girl \\ 2019 Mathical winner, Ages 7-12 \\ Stacy McAnulty \\ Random House 2018}

Unlike the other books discussed so far, this book is more about the culture and people of mathematics, rather than the mathematics itself. It is a charming story about being different and finding the people around you who will value you. The hero, Lucy Callahan, both loves and is exceptional at mathematics, has been home schooled, and is ready for college. She faces a new adventure first: spending a year in middle school. This produces many challenges, especially as, in addition to the mathematical skills, she is not neurotypical and has several obvious routines, such as needing to sit down three times (the first digit of pi) before she can settle.

There are some nice mentions of mathematics, including that the hero should be "dedicating [her] life to solving the unsolvable problems of Mathematics like the Riemann Hypothesis or Hodge conjecture." There is perhaps a little too much presentation of mathematics as simply doing arithmetic fast. The most developed mathematical idea, however, is a solid model which both fits the story and does not claim to be stronger than it is. It uses the statistics of an animal shelter to estimate the length of time for adoption of different dogs, and thus which dogs most need support to find a home.

There is a lot to admire in this story: the presentation of neurodiversity is both gentle and understanding, and the hero is a girl proudly capable in mathematics. There is a problem, however, in combining the two. Lucy's nickname, "Lightning Girl," alludes to a time that she was struck by lightning, the event that "rewired [her] brain transforming [her] into Lucille Fanny Callahan, math genius." In addition to the mathematical ability, this event was responsible for her neurodivergence. This is a little troubling as it suggests the trope that mathematical ability is some sort of compensation for a neurodiverse brain (which is problematic for neurodiverse people who do not excel at mathematics). The message is also that mathematical ability is something inherent (in this case placed essentially as a superpower with a superhero origin story) rather than something that can be obtained by hard work. 


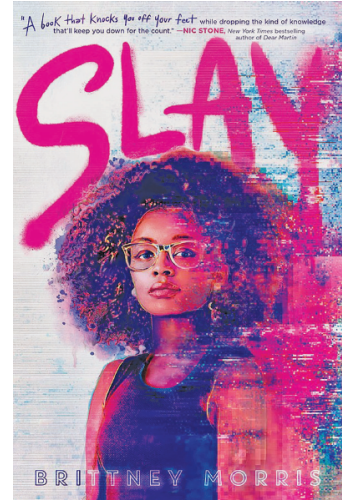

math, but does need to study.

In addition to studying, Kiera Johnson (the hero) is also finding time to develop and administer "Slay," a fantastical online world of virtual reality in which players can create characters and duel in a card game. Games between the best players draw huge crowds. The central focus of Slay is as a space of Black excellence and acceptance, with the game cards each representing some aspect of that culture (both US and international) from twist outs and Gabby Douglas to Louisiana barbeque and Jimi Hendrix's "Purple Haze."

This world is disrupted when a player of the game is murdered and it comes to public attention. This provides the background for examples and discussion of different ideas of Black excellence and racism, with Kiera's mathematical and programming ability playing a central role, whether she is tutoring her peers, keeping the game balanced with the introduction of new cards, or programming an online world.

Like Baby Goes to Market, this shows a more diverse face to those who can excel at mathematics, though in this case for an older audience. It explores the challenges in the clash of ability, identity, and the beliefs and stereotypes that others have developed, and the desire to create spaces where people can unashamedly be themselves.

\section{Conclusion}

There is a growing literature giving tools to support a path to joy and creativity in mathematics. They often help the mathematics to exist in a contextual world (whether real or pure mathematical imagination), rather than the more direct problem-solving approach of school math textbooks. This allows anyone to cultivate joy, interest, and motivation in mathematics in the young people in their lives. Mathematics can find ways to get hard for everyone, and the more we approach that as a challenge and not a barrier, the easier it is to get through and perhaps even enjoy the process of learning. I wish you luck on your path to passing on that inspiration.

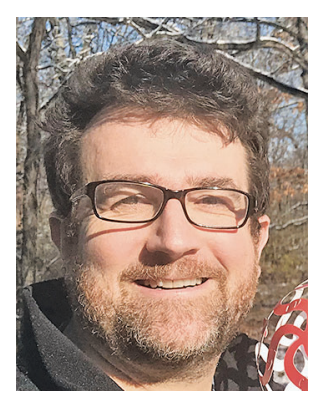

Edmund Harriss

Credits

Cover of Pigeon Math is courtesy of The Innovation Press.

Cover of Slay is courtesy of Simon and Schuster.

Photo of Edmund Harriss is courtesy of Ásgerður Johannesdottir.

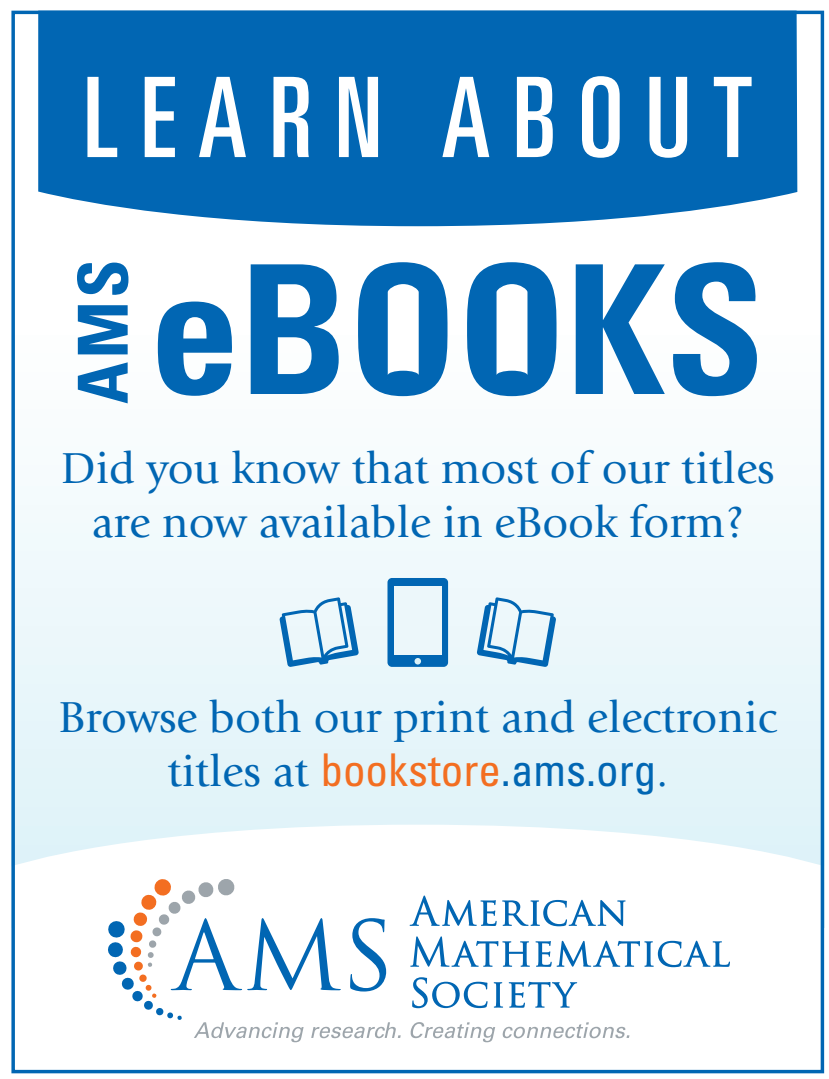

